\title{
FEMTOSECOND ELECTRON BUNCH LENGTHS IN THE SLAC FFTB BEAMLINE*
}

\author{
P. Emma ${ }^{\dagger}$, R. Iverson, P. Krejcik, P. Raimondi, J. Safranek \\ Stanford Linear Accelerator Center, Stanford, CA 94309, USA
}

\begin{abstract}
We describe a new proposal to generate bunches as short as $35 \mathrm{fsec}$ rms with the existing SLAC linac and damping ring. The bunch is compressed in three stages, starting with the existing ring-to-linac compressor. A second stage of compression is added in the form of a simple magnetic chicane installed at the $9-\mathrm{GeV}$ location in the linac. The short bunch after the chicane generates a strong wakefield in the linac which provides a large correlated energy spread allowing a third compression stage in the existing Final Focus Test Beam (FFTB) beamline. Peak currents as high as $30 \mathrm{kA}$ are possible and when passed through a new undulator in the FFTB, can generate hard $x$-rays of very high peak brightness and pulse duration of $80 \mathrm{fsec}$ FWHM. The short bunch is ideal for plasma and wakefield studies as well as providing abundant $R \& D$ for verifying micro-bunch behavior in the future Linac Coherent Light Source (LCLS) [1].
\end{abstract}

\section{INTRODUCTION}

Several proposals [2], [3], [4] exist for generating short bunches using the SLAC linac, but the bunch is typically not short enough, or the location is not ideal (e.g., inside the SLC arcs). We describe an extremely short bunch ( $35 \mathrm{fsec}$ rms) produced in the convenient environment of the existing FFTB hall, at a reasonable cost and effort.

There is a great deal of interest in producing short bunches in the SLAC linac both for experimental applications and accelerator research and development. These range from using short bunches to produce femtosecond spontaneous $x$-ray radiation in an undulator, to wakefield and plasma studies that utilize the extremely high fields associated with the compressed bunch. The technology of producing and diagnosing short bunches in the accelerator is directly applicable to the future success of the LCLS and other accelerators.

The shortest possible bunch produced by the linac with damping ring (DR) is estimated using the conservation of longitudinal emittance.

$$
\sigma_{z_{\min }}=\frac{E_{\mathrm{DR}}}{E_{f}} \frac{\sigma_{\delta_{\mathrm{DR}}}}{\sigma_{\delta}} \sigma_{z_{\mathrm{DR}}}
$$

The minimum bunch length, $\sigma_{z_{\min }}$, is given by the DR energy, $E_{\mathrm{DR}}$, the final energy in the FFTB, $E_{f}$, the relative energy spread at DR extraction, $\sigma_{\delta_{\mathrm{DR}}}$, the extracted bunch

\footnotetext{
* Work supported by DOE contract DE-AC03-76SF00515.

$\dagger$ Emma@SLAC.Stanford.edu
}

length, $\sigma_{\mathrm{zDR}}$, and the final, tolerable energy spread in the FFTB, $\sigma_{\delta}$. The DR energy is $E_{\mathrm{DR}} \approx 1.19 \mathrm{GeV}$, and, with $\sim 2 \times 10^{10}$ electrons per bunch, the rms energy spread is $\sigma_{\delta_{\mathrm{DR}}} \approx 0.08 \%$, and the rms bunch length is $\sigma_{\mathrm{zDR}} \approx 6 \mathrm{~mm}$. With acceleration to $28 \mathrm{GeV}$ and FFTB optics which can tolerate an energy spread of $1.5 \%$, the rms bunch length is limited to $\sigma_{z_{\min }} \approx 12 \mu \mathrm{m}$. The compression must be linear in order to achieve this limit and the compression systems must be compatible with respect to PEP-II operations and existing linac and FFTB hardware. The scenario described below achieves this minimum bunch length for the core of the beam, is compatible with PEP-II operations, requires a new four-dipole chicane in sector-10 (1/3 ${ }^{\text {rd }}$ point $)$, and a new sextupole magnet in the FFTB.

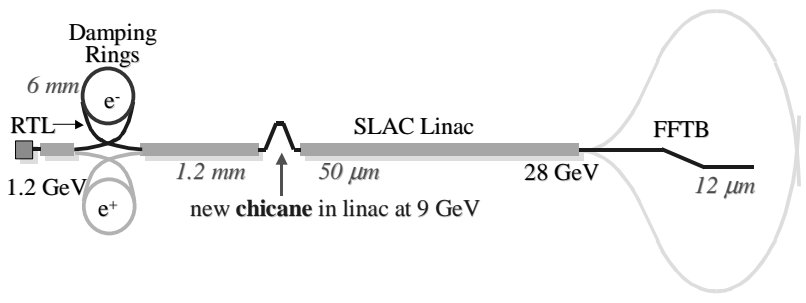

Figure 1. Layout of SLAC linac with 30-kA compression.

Table 1. Electron beam parameters for a 30-kA beam.

\begin{tabular}{|l|c|c|r|}
\hline bunch population & $N$ & 2.2 & $10^{10}$ \\
ring energy & $E_{\mathrm{DR}}$ & 1.19 & $\mathrm{GeV}$ \\
DR rms bunch length & $\sigma_{z \mathrm{DR}}$ & 6.0 & $\mathrm{~mm}$ \\
DR rms energy spread & $\sigma_{\delta_{\mathrm{DR}}}$ & 0.08 & $\%$ \\
final energy & $E_{f}$ & 28.5 & $\mathrm{GeV}$ \\
final rms bunch length & $\sigma_{z}$ & 12 & $\mu \mathrm{m}$ \\
final rms energy spread & $\sigma_{\delta}$ & 1.5 & $\%$ \\
normalized emittances & $\gamma \varepsilon_{x} / \gamma \varepsilon_{y}$ & $50 / 10$ & $\mu \mathrm{m}$ \\
\hline
\end{tabular}

The bunch is compressed in three stages as shown in Figure 1. From a 6-mm bunch length at DR extraction with $2.2 \times 10^{10}$ electrons per bunch, the first stage uses the existing SLC bunch compressor (RTL) to produce a 1.2$\mathrm{mm}$ rms bunch length at $1.2 \mathrm{GeV}$. The second stage is a new four-dipole chicane at $9 \mathrm{GeV}$ in the linac, just after the PEP-II $e^{-}$extraction point. Compression to $50 \mu \mathrm{m} \mathrm{rms}$ is accomplished here with a $1.6 \%$ rms energy spread. This is a 12-meter long chicane with 2-meter bends which may be placed in an acceleration free area at the end of sector-10. The bunch is accelerated in the same linac structures as the PEP II injection beam, on spare machine cycles, without the expenditure of additional RF power. 
The accelerating phase needs to be controlled on these pulses to introduce the necessary energy correlation in the bunches to be compressed. Accordingly, sectors 2 through 10 , from DR to magnetic chicane, are phased at $-20^{\circ}$ from crest. Sectors 11 to 19 , beyond the chicane, are phased on crest. At this point the $28-\mathrm{GeV}$ beam coasts through the last unpowered $1 / 3^{\text {rd }}$ of the linac. The strong longitudinal linac wakefield after the chicane produces a correlated energy spread of $1.5 \% \mathrm{rms}$ at $28 \mathrm{GeV}$. This allows the FFTB to be used as a weak compressor, reducing the bunch length by another factor of four, to about $12 \mu \mathrm{m}$ (FWHM/2.355) for a peak current of $30 \mathrm{kA}$. Table 1 lists the electron beam parameters. Other configurations are also possible (with lower peak current), including reduced charge and/or reduced energy spread.

\section{SYSTEM DESCRIPTIONS}

\subsection{Damping Ring}

The longitudinal emittance from the DR sets the final compression limit. Therefore the extracted bunch length and energy spread are important initial parameters. In the tracking (see below), an asymmetric 6-mm temporal distribution from the DR is used, based on a resistive vacuum chamber impedance $(R \approx 880 \Omega$ ), an RF voltage of $800 \mathrm{kV}$, and $2.2 \times 10^{10} \mathrm{ppb}$ [5]. The bunch distribution at the head rises a bit more steeply than at the tail (head at $z<0$ in Figure 2). The relative energy spread is gaussian with an rms of $0.08 \%$. Measurements exist to confirm these levels [6].
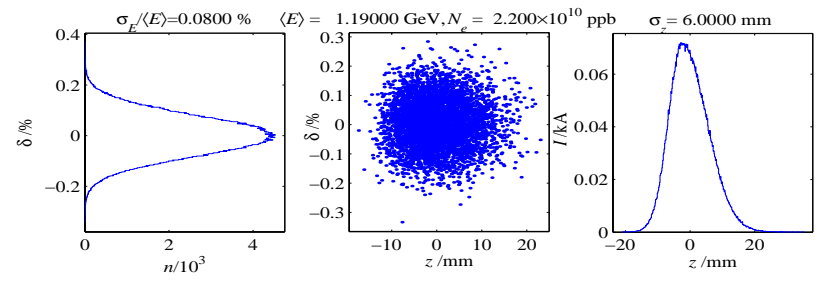

Figure 2. Damping ring longitudinal phase space at $1.19 \mathrm{GeV}$ used for input to tracking studies.

\subsection{Ring-To-Linac (RTL)}

The first stage of compression is the 'RTL' which consists of a short S-band RF section $(2.1 \mathrm{~m})$ and a series of bends $\left(R_{56} \approx 0.6 \mathrm{~m}\right)$ allowing compression from $6.0 \mathrm{~mm}$ to as short as $0.5 \mathrm{~mm}$ rms at $1.19 \mathrm{GeV}$. In order to better shape the temporal distribution after compression, the bunch is over-compressed [7] to a 1.2- $\mathrm{mm}$ rms length. This requires 41-MV of RF at the zero-crossing phase and helps shape the bunch for a more linear, wake-induced energy correlation in the next linac. The large dispersion and energy spread in the RTL generate $2-3 \%$ beam loss.

\subsection{New Chicane Compressor}

The second compression stage is a new four-dipole magnetic chicane installed at location ' $10-9$ ' in the linac at
$9 \mathrm{GeV}$. This location puts the chicane downstream of the PEP-II beam take-off points and will then only influence the less demanding scavenger beam (positron production). The chicane adds a $130^{\circ}$ delay requiring RF re-phasing.

The chicane location is critical, since it sets the linac length seen by the 1.2- $\mathrm{mm}$ long bunch from the RTL. This linac length scales the longitudinal wakefield which must be controlled to maintain the nearly linear energy correlation at chicane entrance. The wakefield of this 785-meters of RF structures is the right strength for a 1.2$\mathrm{mm}$ long bunch with a population of $2.1 \times 10^{10}$ (after $3 \%$ RTL charge loss). A different charge (>5\%) will compromise the linear energy correlation at $9 \mathrm{GeV}$ and limit the final bunch length achievable. The charge can be changed, but to restore linearity, the RTL voltage must also be adjusted, which will alter the bunch length and rescale the wakefield. Charge and other pulse-to-pulse jitter sensitivities are examined below.

A chicane energy of $9-\mathrm{GeV}$ is chosen to conveniently match the PEP-II $e^{-}$energy at this point. Bend and drift lengths are designed to keep synchrotron radiation effects small. With 2-meter long bends and $6.3^{\circ}$ bend angles, the bend-plane emittance growth due to incoherent synchrotron radiation (ISR) is $3.4 \% \quad\left(\gamma \varepsilon_{x_{0}}=50 \mu \mathrm{m}\right)$. Estimates of emittance growth due to coherent synchrotron radiation (CSR) are 5-10\% [8]. Experimental confirmation will be valuable for LCLS R\&D. The $50-\mu \mathrm{m}$ bunch length after the chicane will be measured using the installed transverse RF deflector in linac sector-29 with a voltage $\geq 15 \mathrm{MV}$ [9]. Table 2 lists chicane parameters.

Table 2. Parameters of new 9-GeV chicane.

\begin{tabular}{|l|c|c|r|}
\hline final rms bunch length & $\sigma_{z}$ & 50 & $\mu \mathrm{m}$ \\
rms energy spread & $\sigma_{\delta}$ & 1.6 & $\%$ \\
$R_{56}$ & $R_{56}$ & -75 & $\mathrm{~mm}$ \\
dipole bend angle & $|\theta|$ & 6.33 & $\mathrm{deg}$ \\
max. dispersion & $\left|\eta_{\max }\right|$ & 0.41 & $\mathrm{~m}$ \\
length of each bend & $L_{B}$ & 2.0 & $\mathrm{~m}$ \\
total length of chicane & $L_{T}$ & 11.8 & $\mathrm{~m}$ \\
\hline
\end{tabular}

\subsection{FFTB Beamline}

The final compressor is the FFTB beamline. Its bends are nominally used (with sextupoles) to chromatically correct a small spot size, but can also be used for weak bunch compression. The large, nearly linear correlated energy spread, generated between chicane and FFTB by the strong wakefield of $1.9-\mathrm{km}$ of RF structures, allows the FFTB to compress the bunch using a very small $R_{56}$ value. In fact, the bunch is so short after the chicane that S-band RF phasing beyond this point does little to control energy spread. The spread is produced solely by the wake, and therefore the amount of compression in the chicane completely controls this energy spread.

The sign of the wake requires an $R_{56}$ opposite in sign from the chicane. Fortunately, this is natural in the FFTB 
and $R_{56}=+2.0 \mathrm{~mm}$ adds the final factor four compression. The nominal FFTB has $R_{56} \approx+1 \mathrm{~mm}$, so the adjustment is trivially accomplished by changing three quadrupole magnet settings (with final dispersion also suppressed).

The large energy spread of up to $1.5 \% \mathrm{rms}$ requires care with chromatic aberrations. To this end, the beam is rematched into the FFTB with much smaller beta functions, and the existing $2^{\text {nd }}$-order dispersion is suppressed with a new sextupole magnet. The chromatic emittance growth is then $<2 \%$ based on tracking. Micro-bunch wakefields in the FFTB due to bellows and resistive-wall effects (at $\eta_{x} \neq 0$ ) have also been estimated as an insignificant contribution to emittance growth. Finally, ISR and CSR effects in the FFTB line are expected to increase the bendplane emittance by $5-10 \%$ [8].

\section{PARTICLE TRACKING RESULTS}

Tracking calculations [10], [11] are run which include first and second order compression factors $\left(R_{56}\right.$ and $\left.T_{566}\right)$, geometric wakefields of RF structures, sinusoidal RF, synchrotron radiation in bends (coherent and incoherent), and all $2^{\text {nd }}$-order optical aberrations. This is performed through $>4000$ beamline elements, from DR extraction to end of FFTB. The longitudinal phase space results are shown in Figure 3 at $28.5 \mathrm{GeV}$ in the FFTB. The bunch length is $25-\mu \mathrm{m}$ FWHM with $30-\mathrm{kA}$ peak current and $1.5 \% \mathrm{rms}$ relative energy spread. The transverse and longitudinal results support the feasibility of the proposal.
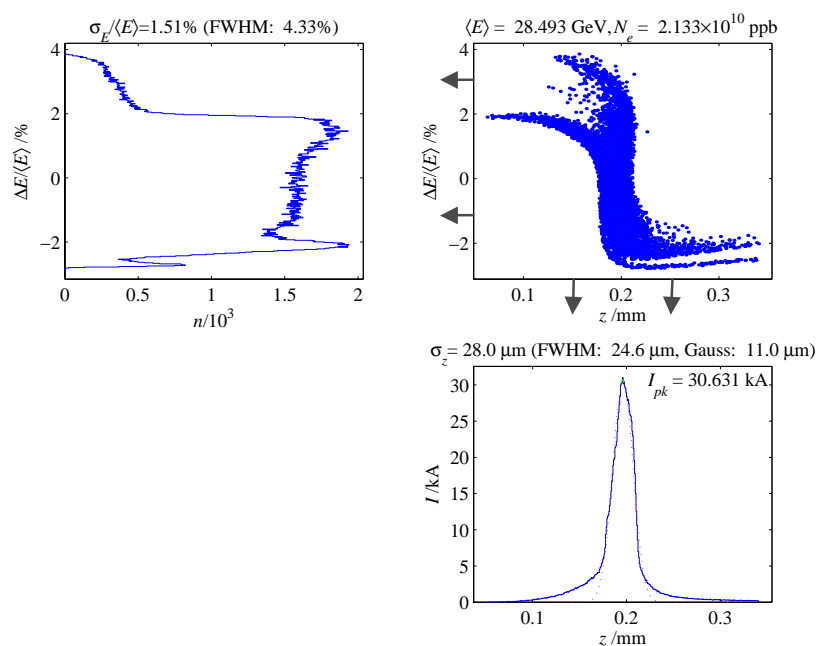

Figure 3. Longitudinal phase space (upper right) at $28.5 \mathrm{GeV}$ after FFTB with distributions (left \& bottom). Bunch population is $2.1 \times 1010$, energy spread is $1.5 \% \mathrm{rms}$, and bunch length is 25 $\mu \mathrm{m}$ FWHM with $11-\mu \mathrm{m}$ rms gaussian fit overlaid in red.

\section{TOLERANCES}

The strong compression imposes tight pulse-to-pulse stability tolerances on RF phase, voltage, and bunch charge. A list of sensitivities is given in Table 3 where each entry causes a $20 \%$ rms change in final bunch length. The tolerances on charge, DR extraction phase, and RF phase jitter are within the observed performance behavior of the SLC [12].

Table 3. Pulse-to-pulse jitter sensitivities for $\left|\Delta \sigma_{z} / \sigma_{z}\right| \leq 20 \%$.

\begin{tabular}{|l|c|c|r|}
\hline bunch charge variation & $\Delta N / N_{0}$ & 7.7 & $\%$ \\
DR extraction phase & $\Delta \varphi_{\mathrm{DR}}$ & 0.9 & deg-S \\
RTL RF phase & $\Delta \varphi_{\mathrm{RTL}}$ & 0.3 & deg-S \\
linac $\langle$ phase $\rangle$ (sec-2 to 10) & $\left\langle\Delta \varphi_{\mathrm{L}}\right\rangle$ & 0.25 & deg-S \\
RF $\langle$ voltage $\rangle$ (sec-2 to 10) & $\left\langle\Delta V_{\mathrm{L}} / V_{\mathrm{L}}\right\rangle$ & 0.8 & $\%$ \\
chicane bend fields (series) & $\Delta B / B_{0}$ & 0.9 & $\%$ \\
\hline
\end{tabular}

\section{CONCLUSIONS}

A peak current of $30 \mathrm{kA}$ is possible in the FFTB beamline using existing SLAC systems. A new chicane is required in the linac and a small sextupole is needed in the FFTB. The LCLS R\&D potential alone is a powerful motivation to carry out this proposal. Short bunch length measurement, stability assessment, CSR verification, and micro-bunch wakefield confirmation are all critical $R \& D$ issues for the LCLS. In addition, $<100$-fsec long hard $x$ ray pulses of $1-\AA$ wavelength can be generated using spontaneous radiation from a short undulator placed in the FFTB hall. The peak spontaneous brightness for a 10meter long undulator can be in excess of $1 \times 10^{25}$ photons $/ \mathrm{s} / \mathrm{mm}^{2} / \mathrm{mrad}^{2} / 0.1 \%$-bandwidth with $\sim 10^{8}$ photons per pulse in a $0.1 \%$ bandwidth.

\section{ACKNOWLEDGEMENTS}

We thank Karl Bane for the damping ring impedance model, and Pisin Chen, Max Cornacchia, Jerry Hastings, Keith Hodgson, and Ron Ruth for encouragement.

\section{REFERENCES}

[1] LCLS Design Study Report, SLAC-R-521, (1998).

[2] J. Seeman, R. Holtzapple, SLAC-PUB-6201, June 1993.

[3] P. Emma, J. Frisch, SLAC-PUB-8308, Dec. 1999.

[4] P. Krejcik, SLAC-PUB-8806, Sep. 2000.

[5] K.L.F. Bane, K. Oide, SLAC-PUB-95-6878, 1995.

[6] K. Bane, et. al., "High-Intensity Single Bunch Instability Behavior in the New SLC DR Vacuum Chamber", PAC95, Dallas, TX, May 1-5, 1995.

[7] F.-J. Decker, R. Holtzapple, T. Raubenheimer, LINAC94, Tsukuba, Japan, August 1994.

[8] M. Borland, "A Simple Method for Simulation of Coherent Synchrotron Radiation in a Tracking Code", ICAP-2000, Darmstadt, Germany, September 2000.

[9] P. Emma, J. Frisch, P. Krejcik, "A Transverse RF Deflecting Structure for Bunch Length and Phase Space Diagnostics", LCLS-TN-00-12, Aug. 29, 2000.

[10] 2D-tracking was performed using the code LiTrack, originally written by Karl Bane, modified by P. Emma.

[11] M. Borland, ICAP-2000, Darmstadt, Germany, September 2000.

[12 ] C. Adolphsen, et. al., "Pulse to Pulse Stability Issues in the SLC", PAC95, Dallas, TX, May 1-5, 1995. 